\title{
Cortes, expertos y grupos de interés: Movilización y localización del conocimiento experto en la sentencia C 355 de $2006^{1}$
}

\author{
Oscar Javier Maldonado Castañeda ${ }^{2}$ \\ Centre for Science Studies, Lancaster University, Lancaster, \\ Reino Unido ${ }^{3}$ \\ o.maldonadocastaeda@lancaster.ac.uk
}

Recibido: 24 de enero de 2013

Aceptado: 25 de junio de 2013

1 Este artículo de reflexión discute algunos de los hallazgos de la investigación "Textualidad, interpretación y construcción del cuerpo: Políticas ontológicas médicas y jurídicas de la despenalización parcial de la Interrupción Voluntaria del embarazo en Colombia" desarrollada entre 2008-2010 para obtener el título de Magister en Sociología de la Universidad Nacional de Colombia. Agradezco las sugerencias hechas por los evaluadores anónimos de este artículo, cuyos comentarios y observaciones lo enriquecieron substancialmente.

2 Sociólogo con Magíster en Sociología de la Universidad Nacional de Colombia. Miembro del Grupo de Estudios Sociales de la Ciencia, la Tecnología y la Medicina de la Universidad Nacional de Colombia.

3 Candidato a Doctor en Sociología y Estudios de Ciencia y Tecnología, Departamento de sociología, Centre for Science Studies, Universidad de Lancaster, Reino Unido. 


\title{
Cortes, expertos y grupos de interés: Movilización y localización del conocimiento experto en la sentencia C 355 de 2006
}

\section{Resumen}

El presente artículo analiza las interacciones entre cortes, expertos y grupos de interés presentes en la sentencia C 355 de 2006, por medio de la cual se despenaliza parcialmente el aborto en Colombia. Una revisión detallada de este texto permite rastrear el rol que tienen grupos de interés y movimientos sociales en la movilización de discursos expertos en las altas cortes como una estrategia para influir en sus decisiones. El uso de disciplinas y campos de conocimiento queda articulado a la estructura general de la sentencia como tecnología literaria que hace visible el papel de la corte como administradora de justicia.

Palabras clave: Experticia, Conocimiento, Autoridad, Amicus curiae, Aborto en Colombia, Altas cortes.

Palabras clave descriptores: Sentencia C-355-2006, Aborto-aspectos jurídicos-Colombia, Derechos del niño, Derechos de la mujer, Colombia. Corte Constitucional.

\section{Courts, Experts and Interest Groups: Mobilization and Location of Expert Knowledge in the Sentence C 355/2006}

\begin{abstract}
This paper analyzes the interactions between courts, experts and interest groups present in the sentence C 355/2006, through which abortion is partially decriminalized in Colombia. A detailed review of this paper allows to track the role that interest groups and social movements have in the mobilization of expert discourses in the high courts as a strategy to influence their decisions. The use of disciplines and fields of knowledge is articulated to the general structure of the sentence as literary technology that makes visible the role of the court as administrator of justice.
\end{abstract}

Keywords: Expertise, Knowledge, Authority, Amicus curiae, Abortion in Colombia, High Courts.

Key words plus: Judgment C-355-2006, Abortion-Legal Aspects-Colombia, Child Rights, Women's Rights, Colombia. Constitutional Court.

\section{Tribunais, peritos e partes interessadas: Mobilização e localização do conhecimento perito na sentença C 355 de 2006}

\section{Resumo}

Este artigo analisa as interações entre tribunais, peritos e partes interessadas presentes na sentença C 355 de 2006, através do qual foi descriminalizado parcialmente o aborto na Colômbia. Uma revisão detalhada deste texto permite rastejar o papel que as partes interessadas e movimentos sociais têm na mobilização de discursos peritos nos altos tribunais como estratégia para influir nas suas decisões. O uso de disciplinas e campos de conhecimento queda articulado à estrutura geral da sentença como tecnologia literária que faz visivel o papel da corte como administradora de justiça.

Palavras-chave: Expertise, Conhecimento, Autoridade, Amicus curiae, Aborto na Colômbia, Altos tribunais.

Palavras-chave descritores: Julgamento C-355-2006, Aborto-aspectos legais-Colômbia, Direitos da criança, Direitos das mulheres, Colômbia. Tribunal Constitucional. 


\section{Introducción}

La sentencia C 355 de 2006, mediante la cual se despenaliza parcialmente el aborto en Colombia, no solo constituye un hito en la reflexión y debate sobre el alcance de los derechos sexuales y reproductivos en el país, sino también es un documento que nos permite rastrear cómo son movilizados argumentos técnicos y jurídicos por diversos actores sociales para influir en las decisiones del Estado. Esta sentencia revela una transición de la esfera pública del debate a círculos más restringidos, donde los grupos de interés tienen un papel muy importante en la movilización de argumentos, discursos y prácticas, mediando la forma en la que entran en escena los expertos, sus discursos y estandarizaciones. Dentro de este litigio, los expertos aparecen adscritos a grupos de interés, salvo contadas excepciones donde aparecen como garantía de neutralidad y objetividad.

La reflexión que presento en este artículo se sitúa dentro de una perspectiva de los estudios sociales de la ciencia dedicada al estudio de las formas de relación entre experticias tecnocientíficas y legales en campos jurídicos (Hilgartner, 1990; Jasanoff, 1994; Edmond, 2001). A través de un acercamiento al recurso jurídico del amicus curiae, este escrito pretende suscitar la reflexión sobre el papel de grupos de interés específicos en el desarrollo de mecanismos de participación de expertos en las cortes.

Asimismo, este trabajo es una contribución al análisis del papel del conocimiento científico y tecnológico en la producción de órdenes jurídicos. En este sentido, un análisis de las formas retóricas y discursivas de interacción de los expertos en campos institucionalizados contribuye a entender cómo se construye la dominación legal y jurídica, así como la creencia en su legitimidad, a través de diferentes mecanismos socio-técnicos ${ }^{4}$.

Finalmente, este escrito pretende, a través del análisis de las relaciones entre derecho y conocimiento tecno-científico, contribuir a la reflexión de lo que implica situar los estudios sociales de ciencia y tecnologia. Situar es hacer explícita la complejidad y la diversidad de los contextos de producción del conocimiento, presentando tanto la materialidad como la historicidad de sus diferentes configuraciones (Haraway, 1997; Barad, 2003). El análisis de objetos jurídicos nos

Siguiendo a Latour (1987) la referencia a lo socio-técnico implica el reconocimiento de la constitución material de lo social, es decir que lo que entendemos cómo lo social adquiere durabilidad y forma a través de la interacción con objetos y tecnologías. 
permite ampliar nuestra comprensión de las configuraciones particulares a través de las cuales se coproducen nuestras experiencias de lo local y lo global, del conocimiento y la política, que definen muchos de los conflictos que han marcado nuestra experiencia de la modernidad en el país.

\section{Despenalización del aborto en Colombia, un contexto}

En Colombia, como en general ha ocurrido en otros países (Baer, 2002) el debate y la efectiva despenalización del aborto, más que ser una iniciativa de los gobiernos, ha sido fruto del trabajo liderado principalmente por grupos feministas y de mujeres, que han abierto el debate a contextos más amplios, como los medios de comunicación, las calles, la academia y las cortes (Gómez, 2006). En Colombia la modernización institucional y política que abre la Constitución de 1991 posibilita la ampliación de la proyección política de estos grupos en un escenario internacional cada vez más sensible a la idea de derechos sexuales y reproductivos (Halfon, 2007).

La controversia en torno a la legalización del aborto en Colombia se remonta a la inserción de tecnologías reproductivas a partir de la década de 1960. La preocupación por el control médico y social de la reproducción y de la sexualidad por parte del Estado situó este procedimiento en un debate entre políticas de control físico y moral agenciadas por grupos modernizantes frente a politicas de control tradicionales como las ejercidas por el Estado bajo la influencia de la Iglesia (Viveros, 1996; Viveros, 2003; Viveros \& Gil, 2006).

Desde la década de 1960 hasta nuestros días se presentaron ocho proyectos de ley y cuatro demandas de inconstitucionalidad que intentaron la legalización o la despenalización del aborto. La última demanda presentada por la abogada Mónica Roa, representante de la ONG Women's link worldwide ${ }^{5}$, consiguió la despenalización de la interrupción voluntaria del embarazo (IVE) en las siguientes circunstancias:

(i) Cuando la continuación del embarazo constituya peligro para la vida o la salud de la mujer, certificada por un médico; (ii) Cuando exista grave malformación del feto que haga inviable su vida, certificada por un

5 Women's Link Worldwide es una organización internacional de derechos humanos, sin ánimo de lucro, enfocada en el área de justicia con perspectiva de género. Fue fundada en 2001, actualmente cuenta con oficinas regionales en Europa (Madrid, España) y Latinoamérica (Bogotá, Colombia). 
médico; y, (iii) Cuando el embarazo sea el resultado de una conducta, debidamente denunciada, constitutiva de acceso carnal o acto sexual sin consentimiento, abusivo o de inseminación artificial o transferencia de óvulo fecundado no consentidas, o de incesto. (C 355 de 2006, p. 301)

La presentación de estas demandas desde la mitad de la década de 1970 coincide con el impacto internacional de la legalización del aborto en Estados Unidos, el caso Roe versus Wade ${ }^{6}$ (1973). En Colombia las estrategias retóricas y jurídicas de los grupos a favor de la despenalización, si bien están inscritas en el discurso del derecho a la elección, no sitúan el problema del aborto como un asunto privado sino como un asunto público. Esto significa que la despenalización del aborto ha sido promovida más como una medida de salud pública -dirigida a reducir tasas de mortalidad de las mujeres que se ven obligadas a recurrir a prácticas riesgosas- que como una reafirmación del derecho a la privacidad y control sobre el propio cuerpo. En las circunstancias de despenalización, se privilegia una mirada médica y experta por encima de la experiencia íntima y personal de la mujer frente a su corporalidad (Maldonado, 2011).

Dentro de los diferentes estudios realizados sobre el aborto en Colombia $^{7}$, es importante destacar algunos análisis de las dimensiones socio-culturales de esta controversia hechos desde una perspectiva feminista y de género. Estos trabajos han enmarcado este problema como un conflicto cultural y político entre visiones tradicionales y modernas del Estado y la sociedad (Viveros, 1996; Dalén, 2011; Jaramillo y Alonso, 2008; MSVM, 2009). Con este fin, se han hecho análisis del discurso de los diferentes grupos de interés involucrados en escenarios de toma de decisión (Viveros, 1996; Dalén, 2011), se ha analizado la posición de grupos profesionales y de expertos en el debate (Viveros, 1996) y el papel de movimientos sociales ${ }^{8}$ en el proceso

6 Roe versus Wade es el título que recibe la sentencia de la Corte Suprema de los EE.UU proferida en 1973 mediante la cual se legaliza el aborto a nivel federal. En este caso la corte reconoce el derecho a abortar como una extensión del derecho constitucional a la privacidad. Esta sentencia constituye un hito en el reconocimiento de derechos sexuales y reproductivos.

7 En relación a los estudios desarrollados sobre el aborto en Colombia, hay un amplio espectro de disciplinas y acercamientos, se han desarrollado decenas de tesis, trabajos de grado, artículos y libros. Estos trabajos son muy diversos, abarcan miradas estrictamente filosóficas, teológicas y éticas (Villarea, 1998; Cotes, 2005), acercamientos médicos y de salud pública (AGI, 2006); también hay otros centrados en las dimensiones económicas y sociales del fenómeno (Gómez, 2006; Gonzales, 2005; Viveros, 1996; 2006; Zamudio, 2000), y muchos más centrados en debates jurídicos (Hoyos, 2005; Jaramillo y Alonso, 2008; Plata, 1997; Velázquez, 2006), tan solo por reseñar algunos de estos trabajos.

8 A diferencia de Jaramillo y Alonso (2008) que presentan la despenalización como el fruto del trabajo de círculos restringidos de expertos altamente calificados en derecho y litigios internacionales, 
de despenalización en Colombia como cambio político (Jaramillo y Alonso, 2008; MSVM, 2009).

En relación al debate jurídico que enmarcó el desarrollo de la sentencia C 355 de 2006, Jaramillo y Alfonso (2008) reconstruyen el litigio a partir de dos estrategias desarrolladas por los grupos de interés detrás de la demanda. La primera estrategia fue el manejo de medios masivos y la segunda, el litigio ante la Corte Constitucional. En relación a la sentencia C 355 de 2006, las autoras, a partir de un análisis de su contenido, describen la transformación de ciertos conceptos que este documento introduce en los marcos constitucionales nacionales. El más importante para ellas es la distinción entre la vida como bien y la vida como derecho, es decir el reconocimiento de la vida del feto como un bien preciado por la sociedad pero la subordinación de este reconocimiento al respeto del derecho fundamental a la vida de la mujer. De esta forma para las autoras, la aparente contradicción que el aborto supondría entre el derecho a la vida de la mujer y del feto queda superada.

Estos trabajos presentan el rol de los diferentes discursos en el desarrollo del debate, así como las continuidades y mutuas influencias entre la esfera pública de los medios de comunicación y los espacios de toma de decisión política (Viveros, 1996; Dalén, 2011). Asimismo, destacan el impacto público de las declaraciones de expertos en medios de comunicación y ante políticos y jueces (MVSM, 2009; Jaramillo y Alonso, 2008). Sin embargo, en estos trabajos el análisis del rol de los expertos se limita a su presencia como figuras de autoridad en la escena pública. Por otra parte, los expertos se identifican únicamente dentro de los grupos en debate, los sectores a favor de la despenalización, como portavoces del derecho internacional, los derechos humanos y la salud pública.

\section{Cortes y saberes expertos}

Dentro del debate sobre aborto en Colombia es claro el uso de discursos expertos por las dos partes de la controversia. En los últimos años ha habido una transformación en la retórica anti-aborto. Como señala Baer (2002), las primeras manifestaciones "eran mucho más

la Mesa por la Salud y la Vida de las Mujeres, MSVM, enfatiza el papel del movimiento social de mujeres como principal actor detrás del litigio y la dificultad de establecer consensos y una movilización conjunta dentro de los diversos grupos sociales dedicados a la equidad de género. 
explícitamente patriarcales y machistas que las contemporáneas". La literatura sobre estudios de ciencia y tecnología desde una perspectiva feminista (Baer, 2002; Isaacson, 1996; Petreski, 1986, Haraway, 1997), ha señalado la existencia de nuevas estrategias retóricas que hacen un complejo uso de una serie de innovaciones tecnológicas, como la ecografia y las distintas técnicas de fotografia médica ${ }^{9}$, resaltando así el impacto de las imágenes fetales y embrionarias en la cultura visual general.

A continuación presentaré un análisis de la figura del amicus curiae, una de las múltiples formas mediante las cuales los grupos de interés movilizan conocimiento experto en su esfuerzo por influenciar la decisión de la Corte. Este estudio se atiene al registro de los amici curiae hechos en la sentencia de la Corte Constitucional de Colombia C 355 de 2006. Mi interés es rastrear cómo los argumentos, tanto a favor como en contra del aborto, traducen repertorios discursivos tomados de las ciencias biomédicas y del derecho de forma estratégica según sus propias preocupaciones. Las dos partes apelan a dos grandes discursos de la modernidad para justificar sus puntos de vista: la ciencia médica (vida) y la democracia (libertad). Cada parte pretende ser la interpretación más auténtica de estos valores y de estos cuerpos de conocimiento. Por esta razón más que asistir a un debate entre grupos modernos contra grupos premodernos o tradicionalistas, vemos la confrontación de dos culturas políticas inscritas en el discurso de la modernidad.

Es importante destacar que este análisis apela a la simetria como un recurso metodológico para rastrear las apropiaciones de diferentes cuerpos de conocimiento experto por las diferentes partes en controversia. La simetría es uno de los principios esbozados por David Bloor (1976) en su Programa Fuerte para una sociología del conocimiento científico. La simetría implica el mismo tratamiento metodológico para las partes de la controversia, independientemente de si el debate se ha cerrado y una de estas es reconocida como cierta o verdadera. Para el tratamiento de esta controversia en particular, la simetría busca facilitar la descripción de aquellas prácticas y discursos que son compartidos por las partes en debate. No se trata de invisibilizar, ni de desconocer las asimetrías de poder, sino por el contrario, este es un esfuerzo por presentar con mayor detalle la naturaleza de estas relaciones a través del análisis de sus múltiples materializaciones, entre ellas el amicus curiae.

9 Ver The Miracle of life, Lennart Nilsson. BBC, el Cuerpo humano, un milagro común. Este documento es un ejemplo de las retóricas de divulgación científicas alrededor de la sobrenaturalidad de lo natural. 
En este contexto los discursos expertos son usados para presentar la controversia como un asunto de hechos, más que como un asunto de valores. Sin embargo, en la sentencia se puede observar que estos elementos se articulan de forma compleja con otras representaciones, incrementando la emotividad del discurso de cada una de las partes.

Los textos que constituyen el núcleo de análisis de este trabajo son documentos jurídicos, estos documentos tienen un aura que hace que se erijan como una presencia material-discursiva de la autoridad de ciertas instituciones dentro del sistema político que da forma al Estado colombiano. En este sentido, el valor de los textos no radica en que sean el registro de acciones y hechos que determinan ciertas situaciones sociales; sino que en sí mismos, los textos y sus codificaciones constituyen uno de los mecanismos mediante los cuales estos poderes operan. La fuerza de un texto jurídico depende de la red legal que moviliza actores, artefactos, cuerpos, grupos sociales e instituciones. En esta cadena de interacciones los documentos legales son un eslabón importante para el reclutamiento de aliados por parte de grupos de interés específicos.

Las sentencias de constitucionalidad de la Corte permiten avanzar en el análisis de los mecanismos retóricos y textuales por medio de los cuales estas instituciones construyen la percepción de justicia. Entre más intereses y grupos sociales antagónicos entren en escena, más complejos serán los recursos usados para que el fallo aparezca como justo, como una manifestación del bien de la sociedad sobre el interés particular.

\section{Litigio público, amicus curiae y la movilización de expertos}

En la década de 1970 surgió en EE.UU. una clase de litigio público centrado en la reivindicación del estatus constitucional de las políticas públicas más que sobre sus consecuencias privadas. Este modelo de litigio ha convertido a diferentes actores jurídicos en protagonistas en el desarrollo de políticas públicas (Sandstrom, 2007). El litigio público ha respondido a asuntos sociales y políticos muy controversiales, abarcando temas como la discriminación racial, las acciones afirmativas, la libertad de expresión, el aborto, las relaciones iglesia-Estado, el derecho a morir, entre otros. Todos estos casos han involucrado una activa e importante participación del amici curiae (Sandstrom, 2007). 
El amicus curiae (literalmente, amigo de la corte) es una figura jurídica mediante la cual "terceros ajenos a un litigio" pueden ofrecer voluntariamente un concepto técnico o jurídico para colaborar con el tribunal en la resolución de la materia objeto de discusión. Si bien esta figura tiene una larga historia dentro de la Common Law, en los últimos años, ha alcanzado un amplio uso internacional vinculado sobre todo al litigio en Derechos Humanos. En este contexto, se ha convertido en una herramienta muy importante para la presentación de conceptos expertos legales y científico-técnicos, afines con las visiones de los grupos de interés que entran en escena. La decisión sobre la admisibilidad de un amicus curiae, generalmente depende de la voluntad del respectivo tribunal. En Colombia este recurso ha sido clave como instrumento de manifestación de ONG's y grupos de interés en materia de Derechos Humanos.

Los grupos de interés que participan como amici curiae persiguen fines políticos (en términos de política pública). Este es el supuesto subrayado por la mayoria de análisis de participación organizada de intereses en la Corte, donde queda abierta la cuestión sobre el impacto de estas declaraciones en sus decisiones (Collins, 2007, p. 56).

En este sentido, la influencia que pueda alcanzar la participación de los amici sigue la jerarquía del sistema de justicia. Si los grupos pueden dar forma a las direcciones ideológicas de las decisiones de la Suprema Corte, directamente afectan la dirección ideológica de las bajas cortes, influenciando todo el sistema de justicia. Esta es una de las razones por las cuales el amicus curiae es una práctica común en las Altas cortes y relativamente rara en otros espacios jurídicos (Collins, 2007, p. 58).

La tensión existente en términos de conocimiento entre campos especializados y el conocimiento jurídico-formal de las Altas cortes es un elemento clave en la comprensión del papel estratégico de los amici curiae. La discontinuidad de competencias (Shapin, 1984) y de poderes introduce y multiplica campos de experticia, las comunidades y los tipos de realidad adscritos a los mismos. La separación de formas de conocimiento, ligada a la capacidad de manipulación de artefactos y teorias, produce formas de dominación y de clasificación social entre expertos y legos, y aún entre los mismos expertos. Hay una brecha entre formas de conocimiento y posiciones de poder. En el caso de toma de decisiones, las instituciones politicas y legales se enfrentan a problemas cada vez más específicos y especializados, a construcciones de alta complejidad (técnica, científica, médica) que 
invocan traducciones y traductores particulares para su comprensión (Jasanoff, 1996; Collins, 2007).

Tal es el caso de las cuestiones legales asociadas con el principio y el final de la vida humana. El debate de estas cuestiones se ha desplazado paulatinamente de los dominios éticos y filosóficos hacia temas como la salud pública y los derechos humanos, llamando la atención de legisladores, proveedores de cuidado en salud y gestores de politica pública (Jasanoff, 1996). Las cortes en estos procesos, renuncian temporalmente a su monopolio sobre las reglas legales concernientes a las tecnologías de prolongación de la vida y las interpretaciones jurídicas de los derechos asociados al inicio y a la terminación de la misma y delegan su control a instituciones de carácter técnico (Jasanoff, 1996).

El proceso de reglamentación de la Sentencia C 355 de 2006, ilustra el punto anterior. El Decreto 4444 de 2006, la Norma técnica de interrupción voluntaria del embarazo (IVE) y las sentencias de tutela T 714 de 2007, T 209 de 2008 y T 388 de $2009^{10}$ recogen el espíritu de la sentencia al dar criterios generales de protección de derechos dejando en manos de los médicos y de procesos técnicos estandarizados los detalles que involucran la definición de las prácticas para cada caso.

La presencia de los expertos no es neutral, menos en estos problemas en donde se ponen sobre la mesa explícitamente las consecuencias sociales, políticas y económicas de determinadas formas de conocer y de actuar sobre la realidad. Los testimonios expertos presentados como amici curiae ilustran las relaciones entre experticias, movimientos sociales y grupos de interés, como veremos a continuación.

10 La sentencia C 355 de 2006 da origen a una red de textos jurídicos en torno al aborto en Colombia. Por ejemplo, el Decreto No. 4444 del 13 de diciembre de 2006 del Ministerio de la Protección Social, por el cual se reglamenta la prestación de unos servicios de salud sexual y reproductiva. Asimismo el incumplimiento de la sentencia por jueces e instituciones prestadoras de servicios de salud ha originado una serie de tutelas que han servido para que la Corte aclare y puntualice los alcances de la sentencia. Las sentencias T 714 de 2007, T 209 de 2008 y T 388 de 2009 abarcan temas como los limites de la objeción de conciencia y la imposición de sanciones a quienes legal o técnicamente obstruyan una IVE. Estas sentencias escenifican de forma intensa los límites impuestos a la mujer, la ambigüedad de las prácticas y los márgenes de cumplimiento de la normatividad jurídica por parte de los diversos grupos de interés, con un agravante: estos procesos ocurren en un marco temporal muy reducido (la gestación). 


\section{Movimientos sociales y conocimiento experto}

Los movimientos sociales han complejizado las relaciones entre ciencia y derecho. Dentro de sus estrategias de actuación política, reconocen que la ley y el Estado son poderosas realidades sociales que no pueden ser ignoradas y que una intervención sistemática sobre las mismas es importante para el alcance de las metas de cualquier grupo de interés organizado (MacKinnon, 1989, p. XIII; Croissant, 2000, p. 228).

En Colombia desde mediados de la década de 1970, se vienen organizando un conjunto de grupos sociales alrededor de la idea de derechos sexuales y reproductivos (Viveros, 2003; Viveros \& Gil, 2006). Este movimiento ha definido la despenalización del aborto como una lucha clave. Una de las caras más visibles de este movimiento en relación con el aborto es la Mesa por la Salud y la Vida de las mujeres, MSVM. Hacia mediados de 2004, alrededor de La Mesa ${ }^{11}$ se debate la posibilidad de despenalizar el aborto vía Corte Constitucional.

La Mesa es un buen ejemplo del papel de los movimientos sociales en la movilización de conocimiento experto (médico y legal) dentro de una estrategia política más amplia. Alrededor de este colectivo se reunieron distintos grupos sociales de activistas y organizaciones técnicas (centros de investigación, universidades, think tanks) para impulsar un debate público sobre aborto en Colombia acompañado de una estrategia de intervención en las Altas cortes (MVSM, 2009).

En la otra orilla de la controversia se encuentra una coalición de iglesias que reúne a la iglesia Católica y otras iglesias cristianas (neopentecostales en su mayoria). La iglesia católica ha recibido particular atención en medios de comunicación y en las cortes, como ha sido usual, la voceria institucional estuvo en manos de los altos jerarcas y de la curia. En las últimas etapas del debate en la Corte fue activa la presencia de organizaciones de laicos y de iglesias cristianas, particularmente a través de marchas y de la presentación de declaraciones ciudadanas y amici curiae.

Los amici curiae constituyen un elemento más del debate que sitúan la controversia dentro de la corte y cuyo objetivo es influir directamente

11 La Mesa por la Salud y la Vida de las Mujeres es un colectivo de instituciones y personas que trabaja para la promoción de los derechos sexuales y reproductivos de las mujeres, en especial, por la legalización del aborto y la práctica del aborto seguro y de calidad en Colombia. Desde 1993, La Mesa por la Salud y la Vida de las Mujeres ha liderado una reflexión continua y sistemática acerca del aborto en Colombia desde variados enfoques, incidiendo de manera directa en el actual proceso de la despenalización parcial. http://www.despenalizaciondelaborto.org.co/page.php?m od $=$ noticies\&idfat $=2 \&$ idsec $=8$ 
en los magistrados a través de la presentación de evidencia. Como se señala en uno de los boletines del Population Research Institute (2005), una de las instituciones más activas en la presentación de amici en contra de la despenalización; presentar evidencia es debatir, es influir a través del conocimiento de manera política. En relación a este litigio se señala que:

(...) Roa se apoya una vez más en las "recomendaciones" del Comité de seguimiento de la CEDAW a las que ella le da un efecto vinculante que en realidad no lo tienen y que según las cuáles Colombia debería modificar las leyes que penalizan el aborto. Roa ha movido sus influencias y ha recogido muchos amicus curiae de todos aquellos que como ella y el Center for Reproductive Rights, verdadera alma mater de Roa, creen que el aborto es un derecho, un "derecho reproductivo" a promover en todo el mundo. Por eso, Colombia necesita de nuestra colaboración. Necesitamos que los magistrados de la Corte Constitucional de Colombia sepan que esas opiniones a favor del aborto no son las únicas ni las más lúcidas. Nosotros sabemos que la razón nos asiste y sólo hace falta que las hagamos llegar oportunamente al sitio donde se necesitan. (PRI, 2005)

A través de los amici curiae, los diferentes grupos de interés movilizan evidencia técnica, científica o jurídica que ellos consideran podría influir en las decisiones de la Corte. A continuación se presenta una descripción de los amici curiae presentados ante la Corte Constitucional tal como quedaron registrados en la sentencia C 355 de 2006. El registro de estas intervenciones ciudadanas hace parte integral de este documento y son un elemento clave en la construcción textual de la distribución de justicia que la Corte proyectó en esta sentencia. Además se hace una breve presentación de dos amici curiae sobre evidencia epidemiológica y de salud pública, los cuales evidencian el rol de este mecanismo en la movilización de ciertas formas de conocimiento y la flexibilidad de su interpretación.

\section{Justicia, conocimiento y textualidad, una mirada a los amici curiae en la sentencia C355 de 2006}

Los textos poseen estructuras para inducir sobre sus lectores una mirada particular, estas, si bien se ven sometidas a la flexibilidad de toda interpretación son tecnologias literarias que construyen formas particulares de credibilidad de los hechos a los que se refieren (Restrepo, 2004). 
Shapin (1984) acuña el concepto de tecnologias literarias para describir el papel que tiene la escritura científica en la construcción de hechos científicos, a través de la figura del lector como testigo virtual.

La tecnología del testimonio virtual involucra la producción en la mente del lector como tal de una imagen de la escena experimental que obvia la necesidad del testigo presencial o de su replicación. A través del testimonio virtual la multiplicación de testigos puede ser en principio ilimitada. Esta fue por tanto la tecnología de constitución de hechos más poderosa. La validación del experimento y la acreditación de sus resultados como hechos, necesariamente supone su realización en el laboratorio de la mente. Para esto lo que se requirió fue una tecnología de confianza y aseguramiento de que las cosas han sido hechas y hechas en la forma descrita. (Shapin, 1984, p. 491)

Las sentencias judiciales y en particular las de las altas cortes, son textos con mecanismos particulares dirigidos a construir confianza en los procesos de deliberación y ante todo en la idea de justicia. Estos documentos poseen tecnologias literarias análogas a las descritas por Shapin (1984) para los artículos científicos. En este caso se invita a cada lector a ser testigo virtual de un hecho social: el funcionamiento del Estado mediante principios racionales y generales donde toda decisión parte de sopesar y escuchar a la sociedad, a los grupos que la constituyen en igualdad de condiciones, para desde el consenso de los magistrados pronunciarse como Corte y administrar justicia. Las sentencias de las altas cortes desarrollan una estructura general y un género literario específico dirigido a la construcción de confianza en la justicia y en su imparcialidad.

Esta finalidad se traduce en una estructura ascendente que va desde las consideraciones de las partes en disputa hacia el concepto y la deliberación de los magistrados. En términos generales una sentencia presenta la siguiente estructura: i. Antecedentes. ii. Normas demandadas. iii. Demandas. iv. Intervenciones ${ }^{12}$. v. Concepto del Procurador General de la Nación. vi. Consideraciones y Fundamentos de la Corte. vii. Decisión. viii. Aclaraciones de voto. La sentencia C 355 de 2006 posee esta misma estructura. En esta se presentan tres demandas de inexequibilidad a la normatividad vigente sobre aborto. A través de la figura

12 Se documentan intervenciones de distintos grupos de interés seleccionados por la corte por su experticia o representatividad frente al problema. Además se incluyen declaraciones e intervenciones que diferentes ciudadanos e instituciones presentan libremente a la corte como contribución al problema. Aqui se presentan los amici curiae. 
de acción pública de inconstitucionalidad se demandaron los artículos 122, 123, 124 y 32 numeral 7 de la Ley 599 de 2000 del Código Penal, los cuales penalizan el aborto en todas las circunstancias.

La demanda construye el problema del aborto en los términos, la lógica y el lenguaje de la corte. La corte entiende los problemas de constitucionalidad en términos de forma y contenido. Aquí, el aborto se presenta como un problema relacionado con la autonomía y el libre desarrollo de la personalidad de la mujer. También como un atentado a su dignidad, vida, salud e integridad. Para el caso de la prohibición del aborto, cuando se han detectado malformaciones en el feto, esta se presenta como un trato cruel, inhumano y degradante. Por otra parte, el problema se construye en términos formales al apelar a principios generales que determinan la justicia del sistema como la proporcionalidad $^{13}$, la subsidiariedad ${ }^{14}$ y la igualdad.

La anterior definición del problema permite que en su tratamiento se apele al derecho internacional, en particular al Derecho Internacional Humanitario, como elemento clave del bloque de constitucionalidad. Como se señala en la demanda presentada por Mónica Roa, consignada en la sentencia C 355 de 2006:

La Corte debe usar como criterio hermenéutico en el estudio de constitucionalidad del manejo penal del aborto la jurisprudencia y doctrina de las instancias internacionales que monitorean los tratados de derechos humanos. De acuerdo a lo anterior, el intérprete debe escoger y aplicar la regulación que sea más favorable a la vigencia de los derechos humanos y para esto debe tener en cuenta la jurisprudencia de instancias internacionales, que constituye pauta relevante para interpretar el alcance de esos tratados. (C 355 de 2006, p. 26)

Este marco interpretativo integra problemas médicos y de salud pública con materias de derecho relacionadas con la equidad de género, la dignidad humana y el libre desarrollo de la personalidad,

${ }^{13}$ El principio de proporcionalidad establece que en la medida de lo posible, debe evitarse el uso desmedido de la fuerza para sancionar infracciones a la ley. En caso de incurrir en estas prácticas, estas deben ser proporcionales al bien que se pretende defender.

14 El principio de subsidiariedad penal, o ultima ratio, establece que si la protección del conjunto de la sociedad puede producirse con medios menos lesivos que los del Derecho Penal, habrá que prescindir de la tutela penal y utilizar el medio que con igual efectividad, sea menos grave y contundente. De esta manera, el Derecho Penal es utilizado como último recurso, exclusivamente para cuando se trate de bienes jurídicos que no puedan ser protegidos mediante el Derecho civil, el Derecho administrativo-sancionatorio (C 355 de 2006). 
situándolos en el mismo plano. Este tipo de construcción del problema permite evadir las cuestiones más tradicionales y polémicas en torno al aborto: el comienzo de la vida humana y el feto como sujeto de derechos.

Después de la definición de los términos de la demanda y su viabilidad constitucional, el texto de la sentencia presenta una serie de intervenciones de distintas entidades, instituciones y ciudadanos que -según la Corte- representarian las voces más autorizadas de la sociedad frente al tema. Las intervenciones se presentan en dos niveles, por una parte aquellas que son directamente invitadas por la Corte Constitucional según la lectura que hace esta de su competencia, legitimidad o representatividad en el tema de discusión; por otra, aquellas intervenciones ciudadanas que libremente y dentro de los tiempos estipulados, se presentan ante la Corte. Estas últimas intervenciones son de especial interés porque si bien apelan al mismo mecanismo de participación pública, son jerarquizadas por la corte según la autoridad y experticia de las mismas.

Las instituciones que la Corte convocó fueron: el Instituto Colombiano de Bienestar Familiar, ICBF, la Corporación Casa de la Mujer, la Corporación Cisma Mujer, el Defensor del Pueblo, el Ministerio de Protección Social, la Conferencia Episcopal Colombiana, la Universidad Santiago de Cali y la Academia Nacional de Medicina. Cada una de ellas, a la luz de la Corte, representaría un sector de la sociedad con legitimidad frente a las diferentes dimensiones del tema. El ICBF representaría el aborto como una materia asociada al cuidado de la familia y de la mujer; la Corporación Casa de la Mujer y Cisma Mujer representarian los derechos de las mujeres; el Defensor del Pueblo participaría desde el punto de vista de los Derechos Humanos; el Ministerio de Protección social participaría desde la salud pública; la Conferencia Episcopal participaría como principal opositora del aborto; la Universidad Santiago de Cali, como expertos en salud pública y la Academia Nacional de Medicina, como autoridad en materia médica.

Posteriormente se referencian las intervenciones ciudadanas, la presencia y la atención concedida a los distintos ciudadanos en el texto de la sentencia están supeditadas a las fechas descritas por la corte para el debate. Las primeras intervenciones ocupan mayor espacio frente a aquellas presentadas extemporáneamente. Las intervenciones están dirigidas tanto a favor como en contra de la despenalización del aborto. La sentencia, en principio clasifica las intervenciones como nacionales e internacionales, a favor de la exequibilidad (en contra 
de la despenalización) y a favor de la inexequibilidad (a favor de la despenalización).

El número de intervenciones ciudadanas en contra del aborto es muy amplio, se presentaron cerca de 1100 frente a 500 que figuran a favor. Este número refleja de forma menos dramática, lo ocurrido en las marchas, según la prensa (El Tiempo, 2006, 14 de mayo), la manifestación antiaborto de 2006 contó con unos 100.000 participantes, mientras las marchas pro-elección no excedieron los 5.000 manifestantes.

Sin embargo, a la hora de caracterizar las intervenciones que la Corte presenta en la sentencia como de expertos, la participación es diferente. Estas contribuciones son el núcleo de lo que se presenta en este ensayo como amici curiae. En primer lugar, el número de intervenciones de expertos a favor o en contra de la exequibilidad es similar. Por otra parte, existe una segregación de áreas de experticia entre los grupos de interés en controversia. Los grupos a favor de la inexequibilidad se concentran en intervenciones en salud pública, derechos sexuales y reproductivos, y legislación internacional. Mientras que los grupos que apoyan la exequibilidad de la penalización del aborto en todas sus circunstancias se concentran en la teoría jurídica, la psicologia y la ética (ver Gráfica 1).

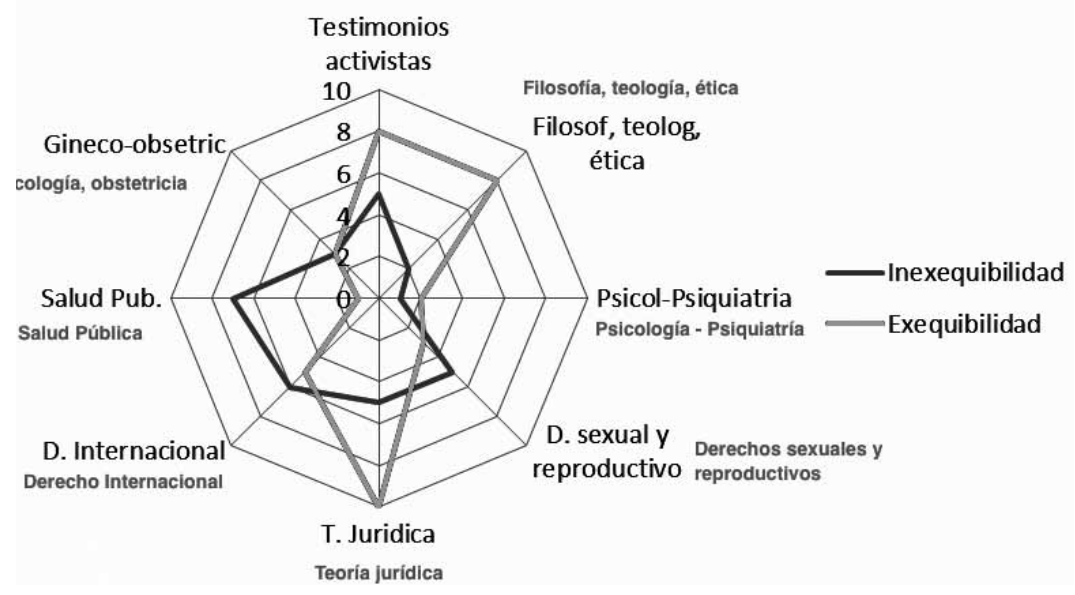

Gráfica 1. Número de intervenciones y amici curiae de expertos en C 355 de 2006 distribuida por áreas de conocimiento.

La concentración en algunas áreas de experticia frente a otras no implica su uso exclusivo por parte de los grupos en controversia. En las diferentes áreas de experticia descritas se encuentran intervenciones 
a favor o en contra del aborto. Frente a la relación directa que se podría establecer entre Teología y prohibición del aborto y entre Derechos sexuales y reproductivos y liberalización del mismo, en el campo de la ética desde una perspectiva teológica es importante la presencia de Católicas por el derecho a decidir, respaldando las demandas de inexequibilidad. En contraste con campos como el de la salud sexual y reproductiva, hay intervenciones en contra de la despenalización como el amicus curiae del Population Research Institute.

El amicus curiae como recurso jurídico enfatiza la discontinuidad de competencias. Este tiene como punto de partida el reconocimiento de las limitaciones de las cortes para fallar sobre el contenido de los asuntos en litigio cuando estos han sido construidos usando repertorios discursivos expertos, especialmente técnico-científicos. Como aparece en el amicus presentado por Católicas por el derecho a decidir ${ }^{15}$ :

Vale la pena notar que el dictamen de la Suprema Corte de los Estados Unidos en el caso de Roe contra Wade exploró esta cuestión con cierto detalle para finalmente concluir que la Corte "no necesita resolver la dificil cuestión sobre en qué momento comienza la vida". $\mathrm{Si}$ aquellos que se han adiestrado en las respectivas disciplinas de medicina, filosofia y teología no han podido llegar a un consenso, en esta etapa del desarrollo del conocimiento humano, las y los representantes del poder judicial no se encuentran en posición de especular sobre la respuesta. (CPDD, 2005, p. 8)

Este amicus advierte sobre la importancia de estos limites en relación al accionar de la Corte que no puede ir más allá de lo que los expertos han logrado en términos de definición de hechos, producidos a su vez por consenso. Sin embargo, los campos de experticia son flexibles, es decir, dentro de su campo existen controversias y debates que permiten que se encuentren instituciones y expertos movilizados a favor, o en contra de los términos en los que se formula determinado fenómeno. Es importante aclarar que hay una tensión entre flexibilidad interpretativa y asimetrías de poder y autoridad dentro de los campos de experticia señalados.

Por otra parte, los campos de experticia son asimétricos en términos de prestigio y autoridad. Existen centros de cálculo (Latour, 1987) en las diferentes áreas. Los expertos adscritos a estas instituciones

15 Este amicus fue presentado por las siguientes instituciones de forma conjunta: Catholics for a free choice, Catholics for a free choice-Canada, Loretto women's network, National coalition of American Nuns, y Women's alliance for theology, ethics and ritual. 
comparten el prestigio y la autoridad de su filiación institucional. La forma como esta autoridad es construida es compleja, responde a procesos históricos, geopolíticos y económicos que posibilitan la construcción de redes entre actores, instituciones y cosas que le permiten a un actor en particular, construir fenómenos de larga escala. Las características de los diferentes actores que intervienen en la construcción de la red que soporta un centro de cálculo influyen en la autoridad y el prestigio del mismo.

A continuación, presentaré brevemente dos amici curiae remitidos a la Corte Constitucional en relación con la despenalización del aborto en Colombia. En primer lugar está el amicus presentado por Alan Guttmacher Institute apoyando la demanda de Mónica Roa y en segundo lugar está un amicus del Population Research Institute en contra de la despenalización. Los dos amici pretenden presentar evidencia epidemiológica y de salud pública en relación al aborto.

El Alan Guttmacher Institute, AGI, es una organización sin fines de lucro dedicada a la investigación, análisis de políticas y educación pública de la salud sexual y reproductiva. Como institución tiene fuertes vínculos con diversos organismos multilaterales, especialmente con la Organización Mundial de la Salud, OMS, y el Fondo de Poblaciones de las Naciones Unidas, UNFPA. Population Research Institute, PRI, es un grupo de investigación sin ánimo de lucro, que tiene por fin "criticar el mito de la sobrepoblación, exponer los abusos a los derechos humanos cometidos en programas de control de población y promover la gente como el mayor recurso del mundo ${ }^{16 "}$. Actualmente sus mayores esfuerzos están concentrados en combatir el aborto a nivel mundial.

Los amici curiae son textos cortos, muchas veces siguen una estructura de preguntas y respuestas y vienen acompañados de textos más extensos como anexos: libros, artículos e informes técnicos. Como recurso, su finalidad es comunicar evidencia soportada principalmente en la autoridad experta y en el prestigio institucional de quienes aparecen como autores de la intervención. En el caso del debate sobre aborto en la Corte Constitucional, estos recursos son primariamente presentados por los abogados involucrados en la controversia, Mónica Roa para el caso de la inexequibilidad e Ilva Myriam Hoyos para el de la exequibilidad, son clave en la presentación de testimonios expertos dentro de sus propias intervenciones. Buena parte de los amici curiae a favor de la exequibilidad de los decretos de penalización del aborto son

${ }_{16}$ Para mayor información, ver: http://www.pop.org/ 
presentados por Ilva Hoyos. De la misma forma que aquellos en contra del aborto son presentados por la Mesa y sus integrantes (Jaramillo \& Alonso, 2008).

Los amici curiae -en tanto textos- soportan las pretensiones de experticia (como lo hacen los textos académicos) con la presencia de un aparato crítico, es decir a través de un sistema de citación y referencias que hace visibles y fácilmente rastreables, las fuentes que soportan sus argumentos. Si bien cada uno de estos institutos tiene una agenda politica, las formas de expresarla y los aliados que cada uno de estos convoca hacen que su prestigio y autoridad dentro de estos escenarios de toma de decisión sean diferentes. En este sentido su capacidad de persuasión depende de su capacidad para traducir sus argumentos en el lenguaje de la corte, en un lenguaje que corresponda a la idea de un Estado secular.

En el caso de los amici presentados por AGI y PRI, contrario a lo que se podría pensar por sus claras divergencias ideológicas, los dos textos comparten una buena cantidad de referencias. Ambos textos usan las mismas fuentes para soportar sus datos, las estadisticas de la OMS e incluso las estimaciones hechas por el mismo AGI. En este caso en particular, se hace explícito un problema de interpretación, de cómo se leen las cifras y se producen relaciones, correlaciones y atribuciones de causalidad. El principal asunto en debate aquí son las consecuencias de la despenalización, tanto en relación a la disminución de mortalidad y morbilidad materna, como respecto a un posible cambio (aumento o disminución) en el número de abortos post legalización.

Por una parte, el AGI señala que:

[...] el problema de la alta mortalidad y morbilidad materna relacionada como consecuencia del aborto en países con leyes de aborto restrictivas ha sido reconocido por la Organización Mundial de la Salud como un grave problema a nivel mundial. Por lo tanto, la tendencia en las últimas décadas ha sido formular leyes menos restrictivas. (AGI, 2005)

En este sentido AGI establece una relación entre barreras de acceso a atención en salud y mortalidad materna, en este caso, contemplando restricciones de tipo legal.

Respecto a este punto y en contraste, el PRI señala que: 
[...] si se hace un análisis comparado de las legislaciones de los países relativas al aborto y las tasas de mortalidad materna, se encuentra que no existe una correlación estadística ni bases científicas para afirmar que a mayor liberalización de la legislación con respecto al aborto exista una disminución de la mortalidad materna. (PRI, 2005)

Para soportar esta afirmación, este amicus presenta las tasas de mortalidad materna de diversos países con diferentes regulaciones respecto al aborto. Este amicus destaca el caso de Rusia y la India, donde el aborto es legal y las tasas de mortalidad materna son altas y el caso de Chile donde hay una regulación restrictiva y unas tasas de mortalidad bajas.

No es la intención de este ensayo evaluar la consistencia metodológica de estas afirmaciones. Sin embargo, es importante destacar el lenguaje empleado para definir la relación entre diferentes eventos; por una parte AGI habla de tendencias y relaciones, mientras que el PRI se remite directamente a correlaciones estadisticas. Estos conceptos plantean diferencias muy grandes en la forma en que se atribuye causalidad y se conectan fenómenos particulares.

A pesar de recurrir a datos similares, la evidencia que se presenta a la Corte por medio del amicus está lejos de ser evidente. En este sentido, más que proveer datos, la función del amicus es proveer un contexto de interpretación e intentar que este sea tenido en cuenta por la Corte. Esto se puede ver en la intervención de PRI, donde información técnica desarrollada por el AGI es interpretada en un contexto completamente diferente:

La legalización del aborto no reduce su incidencia. Contrario a lo que comúnmente se dice, la legalización del aborto no conduce necesariamente a una disminución en las tasas de incidencia. Stanley Henshaw, un reconocido investigador del Alan Guttmacher Institute ha admitido que "en muchos países es común que después de la legalización las tasas de aborto tengan un aumento sostenido por algunos años para luego estabilizarse (AGI, press release, En 6/16/94, PRI, 2005).

Finalmente, un elemento presente en los diferentes amici curiae presentados en este litigio es la presentación de evidencia internacional. La presentación de los datos en el marco de regulaciones y escenarios internacionales es una estrategia presente en los diferentes amicus curiae. Dicha estrategia va en consonancia con la asimilación del derecho internacional dentro del bloque de constitucionalidad que maneja la Corte. 


\section{Efectividad e impacto de los amici curiae}

Volviendo a la sentencia C 355 de 2006, su retórica, en principio inductiva, parte de escuchar a la sociedad expresada en instituciones y ciudadanos libremente movilizados con puntos de vista divergentes y la misma capacidad de participación frente al Estado. Este esquema es clave en la legitimación de los procesos de deliberación de la Corte como asuntos democráticos. En un segundo momento entra en una lógica deductiva, donde unos principios racionales y generales dan luces sobre un caso en particular. Dentro de la estructura de la sentencia se construye una imagen de consenso expresada en la decisión de la corte. Esta se pronuncia como un cuerpo colegiado, que se acoge a la racionalidad del sistema jurídico y vela por su integridad. Hasta aquí en sentido estricto va la sentencia. Sin embargo, el documento cierra con las aclaraciones de voto de los magistrados, en esta parte, los magistrados pueden ilustrar los argumentos que han orientado sus decisiones: aclaraciones cuando han votado a favor de la decisión colegiada de la corte y salvamentos cuando lo han hecho en un sentido contrario.

En esta sentencia se presentan la aclaración de voto del magistrado ponente Jaime Araujo Rentería, la aclaración de voto del magistrado Manuel José Cepeda Espinosa y el salvamento de voto de los magistrados Tafur Galvis, Marco Gerardo Monroy Cabra y Rodrigo Escobar Gil. Vale la pena señalar que las aclaraciones de voto y los salvamentos, si bien son comunes en los documentos de sentencia de la Corte, rara vez son tan detallados y amplios. Hecho que da cuenta de la complejidad del debate y de las precauciones asumidas por la Corte ante el seguimiento de los diversos grupos de interés involucrados en la controversia.

¿Qué opiniones de los amici curiae fueron tomadas en cuenta en esta decisión? Esta cuestión es tan interesante como dificil de responder. Es una pregunta por el impacto y la efectividad de estas intervenciones. En este escrito no me voy a comprometer con una respuesta. Sin embargo, quisiera brevemente proponer una ruta de análisis a través de la visualización de las intertextualidades de los salvamentos de voto. Como presento a continuación, ninguno de los salvamentos hace referencias explicitas a los amici curiae presentados. Sin embargo, dentro de su aparato crítico se usan algunas referencias aportadas por ciertos amici. Estas referencias corresponden principalmente a tratados internacionales y legislaciones sobre aborto de países tomados como punto de comparación. Lo importante aquí más que saber si estas referencias fueron apropiadas de los amici curiae, es ver la afinidad ideológica entre las posiciones de los magistrados 
y los contextos de interpretación propuestos por ciertos grupos de interés, visibles a través de estos documentos.

En relación a los salvamentos y las aclaraciones por una parte, encontramos las aclaraciones de voto de los Magistrados Cepeda y Araujo que sustentan los criterios usados a favor de la inexequibilidad de las normas demandadas. Para Cepeda es fundamental la legislación internacional y el Derecho comparado. En su argumentación cita una importante cantidad de sentencias de altas cortes sobre el tema de diferentes países, principalmente EE.UU, Alemania, Canadá, Italia, Polonia y España. Araujo, por su parte, presenta un espectro amplio de fuentes que van desde textos filosóficos: Aristóteles, Rousseau, Kant, Hegel, West y Berlín; textos de teoría del derecho: Vogel, Dworkin, Novikov, Diez-Picasso, Kelsen y Bobbio; estudios de salud pública: OMS y NIH; hasta una importante selección de sentencias de la Corte Constitucional: C 1039 de 2003, C 371 de 2000, C 1490 de 2000, T 606 de 1995, T 771 de 2000, entre otras. Los textos de Cepeda y Araujo comparten referencias al Caso Roe vs. Wade (1973) de la Corte Suprema de los EE. UU., la Corte Constitucional -como cuerpo colegiado- en el momento de dar su fallo en la sentencia C 355 de 2006 también se remite a este caso.

Por otra parte, encontramos los salvamentos de voto de los magistrados Álvaro Tafur Galvis, Rodrigo Escobar Gil y Marco Gerardo Monroy Cabra ${ }^{17}$ donde exponen sus razones para oponerse a la decisión tomada por la corte en su conjunto. Tafur sustenta su punto de vista en precedentes de la Corte: C 133 de 1994, C 1067 de 2003 y T 409 de 1992; Tratados Internacionales: CEDAW y la Convención Americana de DD.HH de San José de Costa Rica. Escobar y Monroy construyen su argumentación alrededor de sentencias de la Corte: C 133 de 1994, C 591 de 1995, C 447 de 1997, C 013 de 1997, C 406 de 1997, T 128 de 2007, T 223 de 1998; tratados Internacionales: CEDAW, Comité de DD.HH, Tratado de Roma y Costa Rica, y textos de divulgación médica sobre la vida prenatal (claramente en contra del aborto). Los salvamentos de voto descritos hacen referencia común a la sentencia C 133 de $1994^{18}$ y a la Convención americana en DD.HH de Costa Rica. Este texto expresa que el cuidado y la defensa de la vida va hasta antes del nacimiento incluyendo la vida del nasciturus ${ }^{19}$, este texto es citado por la Conferencia Episcopal en su declaración.

\footnotetext{
17 Monroy y Escobar Gil presentan un salvamento de voto en conjunto.

18 Esta sentencia declara la exequibilidad de las normas que penalizan el aborto y consagra la defensa de la vida del nasciturus como deber del Estado.

19 Sobre la distinción entre feto y nasciturus ver el tercer apartado.
} 


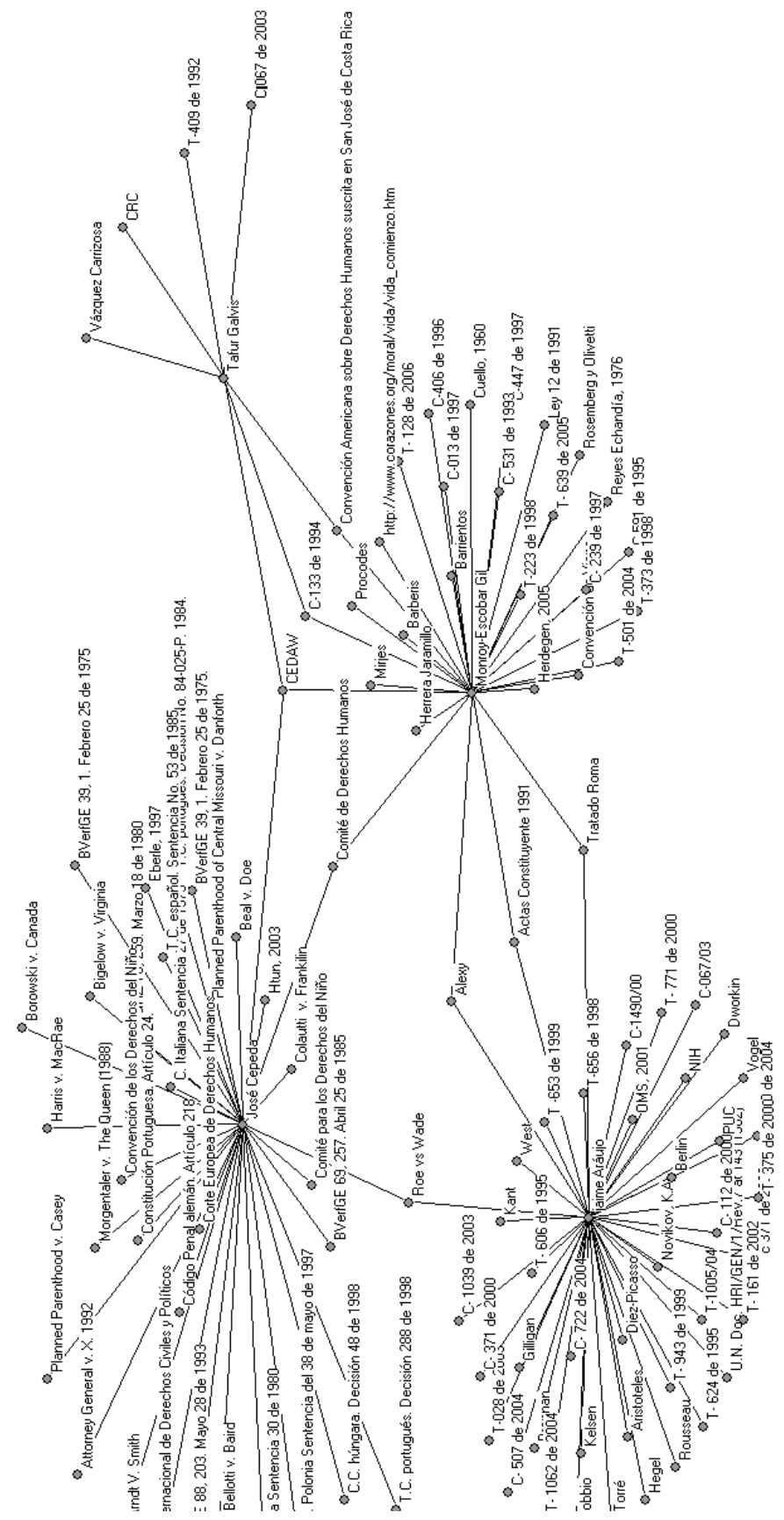

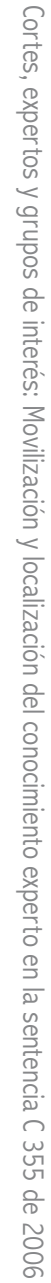

Gráfica 2. Red de citaciones de las aclaraciones y salvamentos de voto de los magistrados en C 355 de 2006. 
En la red de la gráfica 2, se presentan visualmente estas intertextualidades. Los nodos centrales son las declaraciones de los magistrados a los cuales se conectan como nodos periféricos los textos que citan. Este tipo de análisis nos da elementos para rastrear estilos discursivos dentro de las comunidades de expertos, en particular dentro de la Corte Constitucional. Un estilo discursivo es el conjunto de intereses, representaciones, lenguajes y mecanismos retóricos que comparten ciertos individuos sin que necesariamente pertenezcan a un mismo grupo de interés.

El estilo discursivo supone la pertenencia de estos expertos a una subcultura particular dentro de su campo de experticia, esta puede estar mediada por diferentes mecanismos de socialización relacionados con la formación académica, la trayectoria profesional pero principalmente, con los currículos ocultos inscritos en las universidades, historia de vida y las instituciones de trabajo. Los amici curiae como intervención estratégica apuntan a estos estilos discursivos, ellos buscan más que convencer, reforzar ciertas posiciones dentro de la Corte, buscando afinidades técnicas y politicas con ciertos magistrados.

\section{Conclusiones}

La sentencia C 355 de 2006 da cuenta de la complejidad de las relaciones entre experticias en la construcción de objetos públicos y el papel de las textualidadades en este ensamblaje. Alrededor de la despenalización del aborto convergen elementos técnicos múltiples asociados al discurso de la salud pública, la obstetricia y la psicología y cadenas de textos legales, sentencias, declaraciones y litigios de orden local e internacional. Todos estos elementos quedan plasmados en un único texto: la sentencia como registro material del litigio y de los procesos de construcción-legitimación de la justicia.

La sentencia como documento jurídico constituye un punto de partida para la identificación de los diversos grupos de interés que movilizan agendas políticas particulares dentro del Estado y que constituyen un factor dinamizador en el desarrollo de políticas públicas en Colombia. Las Altas cortes han tenido un impacto profundo en la configuración de políticas públicas en el país. El acercamiento detallado a las sentencias de la Corte Constitucional es un elemento clave para entender el rol de elementos regulatorios y legales en el ensamblaje del Estado y en su capacidad de actuación política. 
El análisis de los amici curiae permite identificar el papel de grupos de interés, movimientos sociales y expertos dentro de la tecnología literaria de la sentencia, al ser un elemento clave en la construcción de la legitimidad de las decisiones de la corte y de las formas en que los diversos grupos en controversia segregan algunos campos del conocimiento y se concentran en aquellos que consideran más estratégicos para sus fines específicos.

\section{Referencias}

AGI, Alan Guttmacher Institute. (2005). Amicus curiae presentado a la Corte Constitucional de la República de Colombia.

AGI, Alan Guttmacher Institute. (2006). Preventing Unsafe Abortion and its Consequences: Priorities for Research and Action. New York: Guttmacher Institute.

Baer, J. (2002). Historical and Multicultural Encyclopedia of Women's Reproductive Rights in the United States. Westport, CO: Greenwood Press.

Barad, K. (2003). Posthumanist Performativity: Toward an Understanding of How Matter Comes to Matter, Signs. Journal of Women in Culture and Society, 28 (3), 801-831.

Bloor, (1976/1988). Conocimiento e imaginario social. Barcelona: Gedisa.

CPDD, Católicas por el Derecho a decidir. (2005). Amicus curiae presentado a la Corte Constitucional de la República de Colombia.

Collins, P. (2007). Lobbyists before the U.S. Supreme Court: Investigating the Influence of Amicus Curiae Briefs. Political Research Quarterly, 60 (1), 55-70.

Cotes, M. (2005). La despenalización del aborto en Colombia: una solución innecesaria. Persona y Bioética, 25, 88-100.

Croissant, J. (2000). Critical Legal Theory and Critical Science Studies: Engaging Institutions. Cultural Dynamics, 12, 223-236.

Dalén, A. (2011). El aborto en Colombia. Cambios legales y transformaciones sociales. Tesis de maestría no publicada. Universidad Nacional de Colombia, Facultad de Ciencias Humanas, Escuela de Estudios de Género. Bogotá, Colombia.

Edmond, G. (2001). Legal Engineering: Contested Representations of Law, Science (And Non-Science) and Society. Social Studies of Science, 32 (3), 371-412.

Faúndes, A. y Barzelatto, J. (2005). El drama del Aborto. En busca del consenso. Bogotá: Tercer Mundo editores.

Franklin, S. (1991). Fetal Fascinations: New Dimensions to the MedicalScientific Construction of Fetal Personhood. En Franklin, S., Lury, C., y Stacey, 
J. (Eds.) Off-Centre: Feminism and Cultural Studies (pp. 190-205). New York: Routledge.

Galison, P. (1997). Image \& logic: A material culture of microphysics. Chicago: The University of Chicago Press.

Gómez, C. (2006). Visibilizar, influenciar y modificar: despenalización del aborto en Colombia, Revista Nómadas, 24. 92-105.

González, A. (2005). La situación del aborto en Colombia: entre la ilegalidad y la realidad, Cuadernos de Salud Pública, 21 (2), 624-628.

Halfon, S. (2007). The Cairo Consensus. Plymouth: Lexington Books.

Haraway, D. (1997). Modest Witness@Second Millenium. FemaleMan Meets OncoMouse. London: Routledge.

Hilgartner, S. (1990). The Dominant View of Popularization: Conceptual Problems, Political Uses, Social Studies of Science, 20 (3), 519-539.

Hoyos, I. (2005). La constitución de las falacias: antecedentes de una sentencia. Bogotá: Temis.

Isaacson, N. (1996). The "FETUS-INFANT": Changing Classifications of In Utero Development in Medical Text, Sociological Forum, 11 (3), 457-480.

Jaramillo, I. y Alfonso, T. (2008). Mujeres, cortes y medios: la reforma judicial del aborto. Bogotá: Universidad de los Andes-Siglo del Hombre editores.

Jasanoff, S. (1994). Science at the Bar. Law, Science and Technology in America. Cambridge: Harvard University Press.

Jasanoff, S. (1996). Research Subpoenas and the Sociology of Knowledge. Law and Contemporary Problems, 59, (3), 119-148-

La Mesa por la Vida y la Salud de las Mujeres, MVSM. (2009). Bogotá: Un derecho para las mujeres: La Despenalización parcial del Aborto en Colombia.

Latour, B. (1987). Science in Action. Cambridge: Harvard University Press.

Maldonado, O. (2011). Textualidad, interpretación y construcción del cuerpo. Políticas ontológicas médicas y legales de la despenalización del aborto en Colombia. Tesis de Maestría no publicada. Departamento de Sociología. Universidad Nacional de Colombia, Bogotá, Colombia.

MacKinnon, C. (1989). Feminism in Legal Education. Legal Education Review, 85 (1), 85-95.

Petresky, R. (1986). Fetal images: The Power of visual culture in the politics of reproduction. En M. Stanworth (Ed.). Reproductive technologies: Gender, motherhood and medicine. Minneapolis: University of Minnesota Press.

Plata, M. (1997). Derechos Reproductivos como derechos Humanos: el caso colombiano. En Cook, R. (Ed.) Derechos Humanos de la Mujeres Perspectivas Nacionales e Internacionales. Bogotá: Profamilia. 
Population Research Institute (2005). Amicus curiae presentado a la Corte Constitucional de la República de Colombia.

Restrepo, O. (2004). Retórica de la ciencia sin "retórica". Sobre autores, comunidades y contextos. Revista Colombiana de Sociología, 23, 251-268.

Sandstrom, L. (2007). An Empirical Study of Amici Curiae in Federal Court: A Fine Balance of Access, Efficiency, and Adversarialism. Review of Litigation, $27,669$.

Shapin, S.(1984). Pump and Circumstance: Robert Boyle's Literary Technology. Social Studies of Science, 14 (4), 481-520.

Velázquez, O. (2006). Constitucional y legalmente, el nasciturus es persona y titular del derecho a la vida. Persona y Bioética, 10 (26), 85-103.

Villarrea, J. (1998). Aspectos ético-médicos del aborto. Revista Colombiana de Obstetricia y Ginecología, 49 (1),8-19..

Viveros, M. (1996). El aborto en Colombia: debate público y dimensiones socioculturales. Bogotá: Centro de Investigaciones sobre Dinámica Social (CIDS), Universidad Externado de Colombia, Centro de Estudios Sociales.

Viveros, M. (2003). Género y salud reproductiva en Colombia. En S. Franco (Ed.). La salud pública hoy (pp. 45-68). Bogotá: Universidad Nacional de Colombia.

Viveros, M. (Ed.). (2006). Saberes, culturas y derechos sexuales en Colombia. Bogotá: CLAM-CES-Instituto de Medicina.

Viveros, M. \& Gil, F. (2006). De las desigualdades sociales a las diferencias culturales. Género, "raza" y etnicidad en la salud sexual y reproductiva en Colombia. En M. Viveros (Ed.). Saberes, culturas y derechos sexuales en Colombia (pp. 87-108). Bogotá: CLAM, Tercer Mundo, Universidad Nacional de Colombia.

Zamudio, L. (2000). El aborto en Colombia: dinámica sociodemográfica y tensiones socioculturales, Revista Derecho del Estado, 8, 45-57. 